\title{
Familial 22q11.2 deletions in DiGeorge/velocardiofacial syndrome are predominantly smaller than the commonly observed $3 \mathrm{Mb}$
}

Adewale Adeyinka, MBBS, PhD, Kimberly J. Stockero, BS, Heather C. Flynn, BS, Cindy P. Lorentz, MS, Rhett P. Ketterling, MD, and Syed M. Jalal, PhD

\begin{abstract}
Purpose: DiGeorge/velocardiofacial syndrome (DG/VCFS) is the most common cytogenetically characterized microdeletion of $22 \mathrm{q} 11.2$ region. In $\approx 90 \%$ of patients, the deletion size is $3 \mathrm{Mb}$, whereas the remaining range from 1.5 to $2.5 \mathrm{Mb}$. The purpose of this study was to test the hypothesis that small deletions may be more easily tolerated in a familial fashion than larger deletions, especially for this syndrome. Method: Sixteen FISH probes designed from bacterial artificial chromosomes (BACs) and P1 artificial chromosomes (PACs) mapped to 22q11.2 were used to determine the deletion sizes in 22 individuals from ten families with familial 22q11.2 deletion detected by standard FISH tests. Result: Seven families had deletions of $<3 \mathrm{Mb}(\approx 1.5 \mathrm{Mb})$ in size and 3 families had the common 3-Mb deletion. The 70\% frequency of smaller sized deletions among this group of patients with familial del(22)(q11.2) is significantly higher than that reported among unselected group of patients with del(22)(q11.2) $(P<0.0001$, Fisher exact test). Conclusion: Familial del(22)(q11.2) are predominantly smaller than the common deletion size of $3 \mathrm{Mb}$, indicating that there may be some underlying mechanisms that favor parent-to-child transmission of smaller deletions in individuals with del(22)(q11.2), therefore, underscoring the need to exclude a familial basis in cases of del(22)(q11.2) smaller than 3 Mb. Genet Med 2004:6(6):517-520.
\end{abstract}

Key Words: familial deletion 22q11.2, predominantly small deletions, DiGeorge/velocardiofacial syndrome

The chromosome 22q11.2 region spans a 7.48-Mb interval that is involved in rearrangements resulting in microdeletions and microduplications that are associated with congenital anomaly syndromes. ${ }^{1,2}$ The most common of these 22q11 rearrangement disorders is DiGeorge/velocardiofacial syndrome (DG/VCFS), which results from an interstitial deletion of 22q11.2 and has an incidence of 1 in 4000 to 1 in $6000 . .^{3-5}$ Most individuals with DG/VCFS have interstitial deletions of $3 \mathrm{Mb}$ (present in $\approx 90 \%$ of patients) of DNA. This common $3 \mathrm{Mb}$ deletion, between the genetic markers D22S427 and D22S308, is bordered at both ends by repetitive sequences of DNA known as low copy repeats (LCRs).,.$^{3,6-9}$ LCRs are now known to be the substrate for nonallelic crossovers that result in a variety of structural chromosome abnormalities, including microdeletions. There is ample evidence that the breakpoints of the common $3 \mathrm{Mb}$ and smaller nested deletions seen inpatients with DG/VCFS occur at and around LCRs. ${ }^{3,6-9}$ Indeed, LCRs are thought to comprise about $10 \%$ of the 22 q11 region and eight LCRs have been located here-a plausible explana-

\footnotetext{
From the Cytogenetics Laboratory Department of Laboratory Medicine and Pathology, Mayo Clinic, Rochester, Minnesota.

Syed M. Jalal, PhD, Cytogenetics Laboratory, Department of Laboratory Medicine and Pathology, Mayo Clinic and Mayo Foundation, Rochester, MN 55905.

Received: March 4, 2004.

Accepted: July 8, 2004.
}

DOI: 10.1097/01.GIM.0000144011.97407.B6 tion for the 22q11.2 region's tendency to be involved in structural rearrangements from mismatches of LCRs. ${ }^{1}$ Deletions (about 10\%) and duplications of 22q11.2 are known to be familial and a family of three affected siblings who inherited a 4 $\mathrm{Mb}$ duplication from their mother has been described by us. ${ }^{2}$ Because it is generally accepted that duplications in the genome are better tolerated than deletions, our hypothesis is that smaller deletions may be tolerated in a familial fashion more easily than larger deletions. To test this hypothesis, 16 FISH probes designed from bacterial artificial chromosomes (BACs) and P1 artificial chromosomes (PACs) mapped to 22q11.2 and molecular analysis based on a panel of 15 short tandem repeat (STR) markers were used to determine the deletion sizes in 22 individuals from 10 families with 22q11.2 deletion established by standard metaphase FISH tests.

\section{PATIENTS AND METHODS}

Individuals for study were selected through a search of the Mayo Genetics System (MGS) clinical database, Mayo Clinic, Rochester, MN. The search included 2419 requests for DG/ VCFS from January 1995 to December 2002. Families with at least two members that tested positive for deletion of 22q11.2, using TUPLE1 probe (a standard metaphase FISH test), and for whom stored cells were available for further analysis for at least one family member were included in the study. Institu- 
tional review boards' approval was received for the search and for further genetic tests on patients' samples.

\section{Molecular cytogenetic analysis}

Sixteen direct-labeled fluorescence DNA probes obtained by nick-translation were designed from BACs and PACs spanning regions centromeric and telomeric to TUPLE1. The search for BACs and PACs in the region of interest on 22q11.2 was accomplished using the UCSC (University of California Santa Cruz) Genome Bioinformatics Home Page (http://www. genome.UCSC.edu). Clones were selected based on their location such that each clone flanked or overlapped the next clone. Stab cultures of the BAC and PAC clones were received from Dr. Bruce Roe, Department of Chemistry and Biochemistry, University of Oklahoma. Clones were plated and propagated immediately upon arrival and glycerol stocks were prepared. Isolation and purification of DNA were performed using the QIAGEN Plasmid Maxi Kit according to manufacturer's instructions. The sequences for the BACs and PACs were obtained from the databases available at the National Center for BiotechnologyInformation(NCBI) (http://www.ncbi.nlm.nih. gov/). After editing out repetitive sequences using the CENSOR server at the Genetic Information Research Institute (http://www.girinst.org), primers were designed for each BAC and PAC clone and unique sequences were amplified by polymerase chain reaction (PCR) to verify the identity of the clone. Nick translation was performed using the Vysis Nick Translation Kit (Vysis Inc, Downers Groove, IL) to produce the fluorescence labeled DNA probes for FISH. Probes were labeled with Spectrum Orange-dUTP or Spectrum Green-dUTP, precipitated, and applied to metaphase cells from normal blood specimens to verify hybridization to the region on $22 \mathrm{q}$ and the lack of hybridization to other sequences. The validated probes were then sequentially applied, as previously described, ${ }^{2}$ to interphase and metaphase cells. To confirm the presence or deletion of any one probe, 10 metaphase cells and 100 interphase cells were scored for signals for that probe-the presence of one signal rather than two indicated deletion (Fig. 1). For each individual studied, the probes that were present in two normal copies served as internal controls (Fig. 1).

\section{RESULTS}

Twenty-two individuals from ten families that satisfied the search criteria were identified from among 226 patients that tested positive for deletion of 22q11.2 (using TUPLE1 probe) over the period of seven years. Two of the families had three members, a parent and two children, whereas the remaining eight had two members each: a parent and a child in seven of the families and two siblings in the eighth (both parents were unavailable for testing).

Thirteen of the 16 FISH probes were informative. Analysis of both interphase and metaphase cells were informative and showed that 14 individuals from seven families had $\approx 1.5$-Mb deletions (material was not available to determine the deletion size in two parents), whereas the six members of the remaining three families had $\approx 3-\mathrm{Mb}$ deletions. There was a preponderance of maternal transmissions among the 9 families for which parents were available for testing with TUPLE1 probe and the deletion size remained the same with vertical transmission (Table 1). The centromeric breakpoint in all cases was similar, occurring between the markers D22S427 and D22S1638, which are the markers bordering the proximal LCR implicated in the common 3-Mb DG/
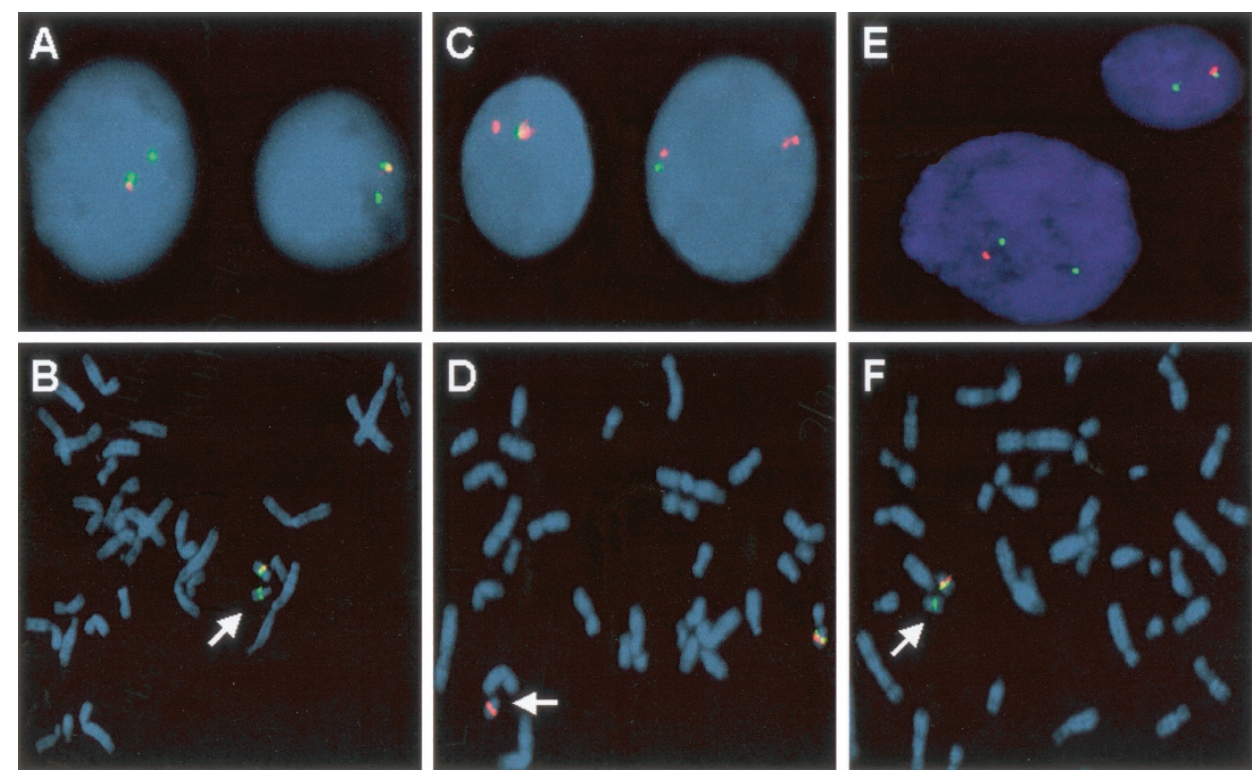

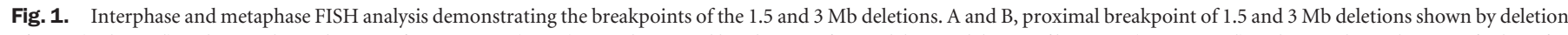

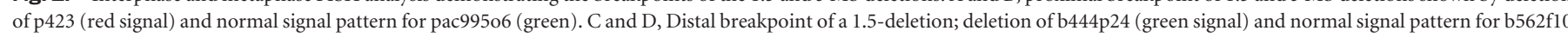

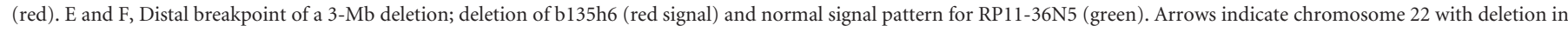
metaphase cells ( B, D, and F). Overlap between green and red signals appears yellow. 
Table 1

Family relationships and individual FISH results of clones on 22q11.2 for 10 families with $\operatorname{del}(22)(\mathrm{q} 11.2)$

\begin{tabular}{|c|c|c|c|c|c|c|c|c|c|c|c|c|c|c|c|c|c|c|c|c|c|c|}
\hline \multirow[b]{2}{*}{ Family } & \multicolumn{3}{|c|}{1} & \multicolumn{3}{|c|}{2} & \multicolumn{2}{|c|}{3} & \multicolumn{2}{|c|}{4} & \multicolumn{2}{|c|}{5} & \multicolumn{2}{|c|}{6} & \multicolumn{2}{|c|}{7} & \multicolumn{2}{|c|}{8} & \multicolumn{2}{|c|}{9} & \multicolumn{2}{|c|}{10} \\
\hline & $\mathrm{P}$ & $\mathrm{C} 1$ & $\mathrm{C} 2$ & $\mathrm{P}$ & $\mathrm{C} 1$ & $\mathrm{C} 2$ & $\mathrm{P}$ & $\mathrm{C}$ & $\mathrm{P}$ & $\mathrm{C}$ & $\mathrm{P}$ & $\mathrm{C}$ & $\mathrm{P}$ & $\mathrm{C}$ & S1 & S2 & $\mathrm{P}$ & $\mathrm{C}$ & $\mathrm{P}$ & $\mathrm{C}$ & $\mathrm{P}$ & $\mathrm{C}$ \\
\hline Age at diagnosis & $31 y$ & $2 y$ & $1 \mathrm{~d}$ & $42 y$ & $22 y$ & $17 y$ & $34 y$ & $10 \mathrm{~d}$ & $55 y$ & $31 y$ & $32 y$ & $2 d$ & $24 y$ & $2 \mathrm{w}$ & ly & $10 \mathrm{~m}$ & $23 y$ & $2 \mathrm{w}$ & $45 y$ & $10 y$ & $40 y$ & $16 y$ \\
\hline Gender & M & $\mathrm{F}$ & M & F & M & $\mathrm{F}$ & $\mathrm{F}$ & $\mathrm{F}$ & F & $\mathrm{F}$ & F & M & $\mathrm{F}$ & M & $\mathrm{F}$ & M & F & M & M & $\mathrm{F}$ & $\mathrm{F}$ & M \\
\hline $\mathrm{b} 476 \mathrm{c} 20(\mathrm{AC} 016027)^{a}$ & + & NT & + & + & + & + & + & + & NA & NT & NA & NT & NT & NT & NT & NT & + & + & + & + & + & + \\
\hline b677f7 (AC008101) & + & NT & + & + & + & + & + & + & NA & NT & NA & NT & NT & NT & NT & NT & + & + & + & + & + & + \\
\hline bac519d21 (AC008079) & + & NT & + & + & + & + & + & + & NA & NT & NA & NT & NT & NT & NT & NT & + & + & + & + & + & + \\
\hline pac995o6 (AC008132) & + & + & + & + & + & + & + & + & NA & + & NA & + & + & + & + & + & + & + & + & + & + & + \\
\hline p423 (AC007326) & - & - & - & - & - & - & - & - & $\mathrm{NA}$ & - & NA & - & - & - & - & - & - & - & - & - & - & - \\
\hline 72f8 (AC000085) & - & - & - & - & - & - & - & - & NA & - & NA & - & - & - & - & - & - & - & - & - & - & - \\
\hline TUPLE1 (HIRA) & - & - & - & - & - & - & - & - & - & - & - & - & - & - & - & - & - & - & - & - & - & - \\
\hline p888c9 (AC005663) & - & NT & - & - & - & - & - & - & NA & NT & NA & NT & NT & NT & NT & NT & - & - & - & - & - & - \\
\hline b444p24 (AC007663) & - & - & - & - & - & - & - & - & NA & - & NA & - & - & - & - & - & - & - & - & - & - & - \\
\hline $562 \mathrm{f} 10(\mathrm{AC} 007731)$ & + & + & + & + & + & + & + & + & NA & + & NA & + & + & + & + & + & - & - & - & - & - & - \\
\hline bac32 (AC007050) & + & + & + & + & + & + & + & + & NA & + & NA & + & + & + & + & + & - & - & - & - & - & - \\
\hline b135h6 (AC002470) & + & NT & + & + & + & + & + & + & NA & NT & NA & NT & NT & NT & NT & NT & - & - & - & - & - & - \\
\hline RP11-36N5 & + & NT & + & + & + & + & + & + & NA & NT & NA & NT & NT & NT & NT & NT & + & + & + & + & + & + \\
\hline RP11-22M5 & + & + & + & + & + & + & + & + & NA & + & NA & + & + & + & + & + & + & + & + & + & + & + \\
\hline Approximate deletion size, $\mathrm{Mb}$ & 1.5 & 1.5 & 1.5 & 1.5 & 1.5 & 1.5 & 1.5 & 1.5 & & 1.5 & & 1.5 & 1.5 & 1.5 & 1.5 & 1.5 & 3 & 3 & 3 & 3 & 3 & 3 \\
\hline
\end{tabular}

P, parent; C, child; S, sibling; y, years; m, months; w, weeks; d, days; M, male; F, female; +, normal signal pattern; -, deletion; NA, sample not available for further tests; NT, not tested.

${ }^{a}$ Clone name and Genebank accession number.

VCFS deletion (Fig. 1 and 2). The telomeric breakpoint for the $1.5-\mathrm{Mb}$ deletions was between markers D22S1623 and D22S264 (Fig. 1 and 2). The LCR implicated in $1.5 \mathrm{Mb}$ DG/VCFS deletions and the constitutional $(11,22)$ translocation lies in this interval. ${ }^{7}$ The telomeric breakpoint for the $3-\mathrm{Mb}$ deletions was between markers D22S1709 and D22S308, which bound the distal LCR implicated in the 3-Mb DG/VCFS deletion (Fig. 1 and 2).

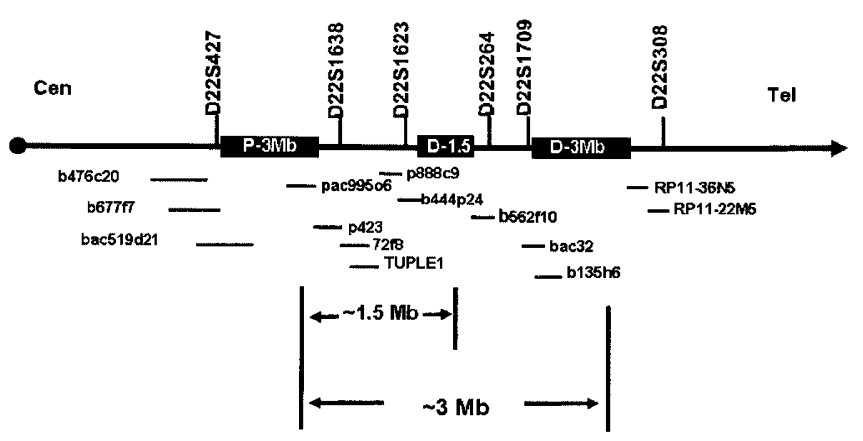

Fig. 2. The DG/VCFS proximal 3-Mb LCR (P-3Mb), distal 1.5-Mb (D-1.5) LCR, and distal 3-Mb LCR (D-3Mb) shown in relation to breakpoints determined by 13 informative BACs and PACs mapping of 22q11.2 in 10 families with del(22)(q11.2). Centromeric breakpoint for the deletions is between pac 99506 and $\mathrm{p} 423$ in all members of the families. Telomeric breakpoint in 14 patients from 7 families with $1.5 \mathrm{Mb}$ deletions is between b444p24 and b562f10. The telomeric breakpoint in 6 patients from 3 families with $3 \mathrm{Mb}$ deletions is between b135h6 and RP11-36N5.

\section{DISCUSSION}

Approximately $90 \%$ of patients with del22q11.2 that are well characterized have a $3-\mathrm{Mb}$ deletion, whereas the remaining have between $1.5-$ to $2.5-\mathrm{Mb}$ sized deletions. ${ }^{5,6}$ Nonetheless, patients with duplication 22q11.2, the reciprocal event of $\operatorname{del}(22)(q 11.2)$, have been shown to have duplication sizes ranging from 3 to $6 \mathrm{Mb}^{2}$ This difference would be in keeping with the expectation that duplications are generally better tolerated than haploinsufficiency. Indeed, the cytogenetic database shows that $11 \%$ of possible autosomal bands were not involved in deletions, whereas only $\approx 2 \%$ were not involved in duplications, implying that there were far more tolerable duplication events in the genome than there were deletions. ${ }^{11,12}$ Extrapolating this concept, one could hypothesize that small deletions would be better tolerated than large deletions. In the present study, we detected a $70 \%$ frequency of deletions smaller than $3 \mathrm{Mb}$ among families with $\operatorname{del}(22)$ (q11.2). This is significantly higher $(P<0.0001$, Fisher exact test $)$ than what is documented in the literature among unselected cases of 22q11.2 deletion..$^{5,6,13}$ In addition the frequency of smaller deletions in the present cohort is higher than that reported among 24 families with inherited $\operatorname{del}(22)$ (q11.2), three of whom had smaller deletions. ${ }^{10}$

Although studies have shown that there is no correlation between the phenotype and size of 22q11. 2 deletions, ${ }^{14}$ a plau- 
sible explanation for the ease of transmitting a $1.5-\mathrm{Mb}$ deletion compared to a 3-Mb deletion would be that deletions, especially larger deletions, have a more deleterious effect when inherited. Indeed, congenital heart defects, a major cause of mortality in DG/VCFS patients is reportedly more severe in offsprings than in transmitting parents. ${ }^{15}$ Furthermore, haploinsufficiency of TBX1, a gene within the $1.5-\mathrm{Mb}$ deleted region, is thought to be a major determining factor for many of the features $\operatorname{del}(22)(\mathrm{q} 11.2)$, whereas other genes like HIRA, UFDIL, also within the 1.5-Mb region, and CRKL (outside of the $1.5-\mathrm{Mb}$ deleted region but within the $3-\mathrm{Mb}$ region) are regarded as possible modifier genes. ${ }^{14} \mathrm{~A}$ 3-Mb deletion would in effect result in haploinsufficiency of more modifier genes than would a $1.5 \mathrm{Mb}$ deletion leading one to speculate that haploinsufficiency of certain modifier genes outside of the $1.5-\mathrm{Mb}$ region makes a $3-\mathrm{Mb}$ deletion more deleterious in embryos inheriting this larger deletion. Studies detailing reproductive outcomes in individuals with $\operatorname{del}(22)$ (q11.2) are necessary to understand how deletion sizes may impact on reproductive capabilities.

Similar to previous observations of 14 transmitting female parents among twenty families, ${ }^{10} 61$ transmitting female parents among 81 families, ${ }^{15}$ and 10 transmitting female parents among 13 families, ${ }^{16}$ we also observed a predominantly female to offspring transmission of inherited $\operatorname{del}(22)(\mathrm{q} 11.2)$ - seven of nine parents were female (Table 1). The importance of this is, however, not clear and we are not aware of any study documenting a sex bias in the severity of phenotypes among individuals with $\operatorname{del}(22)$ (q11.2) that might give female patients a reproductive advantage over males. Nonetheless, no differences were found in the clinical features of patients with DG/ VCFS based on the gender of transmitting parent. ${ }^{10}$

It is estimated that the incidence of familial $\operatorname{del}(22)$ (q11.2) is $\approx 10 \%$ (range $6 \%-14 \%)^{10}$ and the ideal is to exclude the possibility of an inherited deletion in all cases of cytogenetically proven del(22)(q11.2). However, the algorithm for achieving this differs from center to center. Most centers tend to pursue testing in parents who are suspected of having DG/VCFS and less so in parents without overt medical problems or learning disabilities. ${ }^{10}$ Our findings support the hypothesis that deletions that are smaller than $3 \mathrm{Mb}$ are much more common as a familial disorder making it especially pertinent to exclude a familial basis in all cases of $\operatorname{del}(22)(\mathrm{q} 11.2)$. This can be achieved using a FISH test without the need for a standard chromosome analysis. Deletion sizes of $3 \mathrm{Mb}$ or less seem to play a role in the familial transmission of 22q11.2 and although reciprocal duplications of 4-6 Mb have been documented, no deletions of such sizes have ever been reported. Therefore, the mechanisms of selection against large deletions, including spontaneous abortions may be worth evaluating.

\section{References}

1. Spiteri E, Babcock M, Kashork CD, Wakui K, Gogineni S, Lewis DA et al. Frequent translocations occur between low copy repeats on chromosome 22q11.2 (LCR22s) and telomeric bands of partner chromosomes. Hum Mol Genet 2003;12:1823-1837.

2. Ensenauer RE, Adeyinka A, Flynn HC, Michels VV, Lindor NM, Dawson DB et al. Microduplication 22q11.2, an emerging syndrome: clinical, cytogenetic, and molecular analysis of thirteen patients. Am J Hum Genet 2003;73:1027-1040.

3. Shaffer LG, Lupski JR. Molecular mechanisms for constitutional chromosomal rearrangements in humans. Annu Rev Genet 2000;34:297-329.

4. Botto LD, May K, Fernhoff PM, Correa A, KC, Rasmussen SA et al. A populationbased study of the 22q11.2 deletion: phenotype, incidence, and contribution to major birth defects in the population. Pediatrics 2003;112:101-107.

5. Kato T, Kosaka K, Kimura M, Imamura S, Yamada O, Iwai K et al. Thrombocytopenia in patients with $22 \mathrm{q} 11.2$ deletion syndrome and its association with glycoprotein Ib-beta. Genet Med 2003;5:113-119.

6. Carlson C, Sirotkin H, Pandita R, Goldberg R, McKie J, Wadey R et al. Molecular definition of 22q11 deletions in 151 velo-cardio-facial syndrome patients. Am J Hum Genet 1997;61:620-629.

7. Edelmann L, Pandita RK, Spiteri E, Funke B, Goldberg R, Palanisamy N et al. A common molecular basis for rearrangement disorders on chromosome 22q11. Hum Mol Genet 1999;8:1157-1167.

8. Edelmann L, Pandita RK, Morrow BE. Low-copy repeats mediate the common 3-Mb deletion in patients with velo-cardio-facial syndrome. Am J Hum Genet 1999; 64:1076-1086.

9. Shaikh TH, Kurahashi H, Emanuel BS. Evolutionarily conserved low copy repeats (LCRs) in 22q11 mediate deletions, duplications, translocations, and genomic instability: an update and literature review. Genet Med 2001;3:6-13.

10. McDonald-McGinn DM, Tonnesen MK, Laufer-Cahana A, Finucane B, Driscoll DA, Emanuel BS et al. Phenotype of the 22q11.2 deletion in individuals identified through an affected relative: cast a wide FISHing net! Genet Med 2001;3:23-29.

11. Brewer C, Holloway S, Zawalnyski P, Schinzel A, Fitzpatrick D. A chromosomal deletion map of human malformations. Am J Hum Genet 1998;63:1153-1159.

12. Brewer C, Holloway S, Zawalnyski P, Schinzel A, FitzPatrick D. A chromosomal duplication map of malformations: regions of suspected haplo- and triplolethalityand tolerance of segmental aneuploidy-in humans. Am J Hum Genet 1999;64:17021708 .

13. Saitta SC, Harris SE, Gaeth AP, Driscoll DA, McDonald-McGinn DM, Maisenbacher MK et al. Aberrant interchromosomal exchanges are the predominant cause of the 22q11.2 deletion. Hum Mol Genet 2004;13:417-428.

14. Yamagishi H, Srivastava D. Unraveling the genetic and developmental mysteries of 22q11 deletion syndrome. Trends Mol Med 2003;9:383-389.

15. Ryan AK, Goodship JA, Wilson DI, Philip N, Levy A, Seidel H et al. Spectrum of clinical features associated with interstitial chromosome 22q11 deletions: a European collaborative study. J Med Genet 1997;34:798-804.

16. Desmaze C, Prieur M, Amblard F, Aikem M, LeDeist F, Demczuk S et al. Physical mapping by FISH of the DiGeorge critical region (DGCR): involvement of the region in familial cases. Am J Hum Genet 1993;53:1239-1249. 